\title{
High Flow Nasal Cannula Oxygen In Non-Critical Care Units In Patients Infected With SARS-CoV-2 Who Are Not Suitable For Intensive Care Unit
}

Laura Bouetard ( $\square$ laura.bouetard@aphp.fr)

APHP: Assistance Publique - Hopitaux de Paris https://orcid.org/0000-0002-9562-1138

\section{Dorothée Vignes}

APHP: Assistance Publique - Hopitaux de Paris

Dorra Braham

APHP: Assistance Publique - Hopitaux de Paris

Raluca Sterpu

APHP: Assistance Publique - Hopitaux de Paris

\section{Charles Damoisel}

APHP: Assistance Publique - Hopitaux de Paris

\section{Florence Doucet-Populaire}

APHP: Assistance Publique - Hopitaux de Paris

Christelle Guillet-Caruba

APHP: Assistance Publique - Hopitaux de Paris

Mathieu Mion

APHP: Assistance Publique - Hopitaux de Paris

Cécile Goujard

APHP: Assistance Publique - Hopitaux de Paris

Sophie Abgrall

APHP: Assistance Publique - Hopitaux de Paris

\section{Research}

Keywords: COVID-19, High-flow oxygen, elderly

Posted Date: September 17th, 2021

DOI: https://doi.org/10.21203/rs.3.rs-859472/v1

License: (c) (1) This work is licensed under a Creative Commons Attribution 4.0 International License.

Read Full License 


\section{Abstract}

\section{Background:}

High-flow nasal cannula (HFNC) is a recent respiratory support technique used for patients with hypoxemic respiratory failure; its use usually takes place in critical care wards. During the second wave of Covid-19, almost 400000 people were hospitalized in France, and intensive care units were overwhelmed. For patients who did not meet criteria for admission to an intensive care unit because of their age or their medical background, we proposed HFNC in non-critical care unit instead of standard oxygen therapy to improve patient survival and comfort.

\section{Objectives:}

To describe characteristics and outcomes of patients infected with SARS-CoV-2 with HFNC in non-critical care wards between September 2020 and June 2021.

\section{Methods:}

This was a single-center, retrospective cohort study conducted between September and June 2021 in Clamart Hospital, France. Patients infected with SARS-CoV-2 confirmed, who were not suitable for intensive care unit escalation, and who were proposed HFNC because of respiratory failure were assessed.

\section{Results:}

Thirty-one patients with SARS-CoV-2 were included, median age 87.0 years (interquartile range (IQR), 82.0-91.0), 52\% men. Nineteen (61\%) patients were OMS score 0, i.e. no disability in daily-life activities. HNFC was started a median of 3 days (IQR, 1-5.5) after hospitalization. Overall, median duration of HNFC was 6 days (IQR, 4-10). Eleven patients (35.5\%) survived and were discharged from hospital.

\section{Conclusions:}

Our experience of HFNC for patients with COVID-19 outside of a critical care environment because of their age and comorbidities is positive allowing survival of $35 \%$ of old patients not admitted to an intensive care unit.

\section{Trial registration:}

Not applicable

\section{Background}

COVID-19 waves outbreak in France from September 2020 to May 2021 resulted in nearly 373000 hospitalizations and 62993 in-hospital deaths (17\%). The beds occupancy rate in intensive care unit 
(ICU) exceeded 100\% for more than 2 months between March and May 2021 in Paris area.

Overall hospital mortality was lower at the end of the second wave in December 2020 than at the start of the first wave in March 2020 due to improved medical care. However, the probability of death was higher during the second wave than at the end of the first wave. Since mortality outside ICU was significantly correlated with percentage of ICU occupancy, one hypothesis was that increasing pressure on the healthcare system prevented hospitals to correctly deal with the increasing demand. However, proportion of patients that entered ICU was very low among those older than 70 (1).

Due to the high mortality rate in old hospitalized COVID-19 patients who are not suitable for intensive care unit escalation or invasive ventilation (2), we decided to use high flow nasal cannula (HFNC) in the infectious diseases ward. HFNC is a respiratory support, which delivers high flow, heated and humidified controlled concentration of oxygen via the nasal route. This technique, usually delivered in ICU, was developed during the first wave for COVID-19 and sometimes proposed into conventional units as an alternative to invasive mechanical ventilation to postpone ICU admission. We hypothesized that this technique could be useful for the oldest people to try to increase their survival outside ICU or at least to improve their comfort during the oxygen support before death.

The aim of this work was to describe our experience with HFNC in old people in a non-critical care unit.

\section{Methods}

The study was conducted at the Antoine Beclere University Hospital, located in the South-West of Paris area, France, a part of the Assistance Publique-Hôpitaux de Paris, which allows the use of routinely collected data for non-interventional research (https://www.aphp.fr/protection-des-donneespersonnelles). During the whole epidemic, a decision on fitness for invasive ventilation was taken for each patient at the time of admission in collaboration with critical care team, after consideration of age and comorbidities which are associated with high mortality rates and high likelihood of poor outcomes of intensive care in COVID-19 $(1,2)$.

Between September 2020 and June 2021, all patients infected with SARS-CoV-2 confirmed by a positive reverse transcriptase polymerase-chain-reaction (RT-PCR) from a nasopharyngeal swab, with a severe acute lung disease leading to respiratory failure, who were not suitable for ICU because of age and comorbidities were proposed HFNC. Because HFNC need full acceptance of the technique, people with cognitive disorders were not proposed.

Setting up the machine at initiation was with a gas flow rate of $60 \mathrm{~L} / \mathrm{min}$ and a fraction of oxygen $\left(\mathrm{FIO}_{2}\right)$ of 1.0 with a nasal cannula suitable size. High-flow oxygen was continued until it could be switched to standard oxygen therapy after a progressive decrease in gas flow rate to $40 \mathrm{~L} / \mathrm{mn}$ and in $\mathrm{FIO}_{2}$ to 0.4 was possible with oxygen saturation maintained to more than $95 \%$ or until death. 
Demographic and clinical data were retrieved from medical records. Details of SARS-Cov2 infection (chest computed tomography (CT) scan lesions), medical prescriptions were collected. The frailty level prior to COVID and hospital admission was assessed using the clinical frailty scale (CFS) which defines nine classes from very fit to terminally ill, patients with a CFS of 1-3 classified as fit, those with a CFS of 4 as vulnerable and a CFS 5 or higher as frail (supplementary Table) (3).

The primary outcome was the survival rate.

\section{Results}

From September 2020 to June 2021, 333 patients with COVID-19 were admitted to the Infectious Disease unit and 31 (9\%) older than 70 were eligible for HFNC outside ICU. Clinical details and outcomes are shown in Table.

Median age was 88 (IQR, 82-91). Twenty-eight patients (90\%) were living in their personal residence before admission, 9 (29\%) were fit, ie. CFS 1-3. Median body mass index was 25 (IQR, 22-29). Arterial hypertension and chronic cardiovascular diseases (ischemic heart disease, heart failure, atrial fibrillation, pacemaker...) were the most frequent recorded comorbidities. The extent of chest CT-scan lesions was higher than $25 \%$ (major damages) in 13 patients (42\%).

All patients were given standard of care with antipyretic therapy, prevention of thrombotic complications and dexamethasone $6 \mathrm{mg}$ per day for ten days (4). Fifteen (48\%) received intravenous infusion of tocilizumab (antihuman interleukin (IL)- 6 receptor antibody) $8 \mathrm{mg}$ per kilogram at the time of severe increase in their oxygen need, repeated 12 to 24 hours later (5), among whom 6 survived, 9 died.

HNFC was started a median of 3 days (IQR, 1-6) after hospital admission and a median of 3 days (IQR, $1-5)$ after the initiation of conventional oxygen therapy. At HNFC initiation, median oxygen support needed was 12L/min (IQR, 8-15), median arterial oxygen saturation 95\% (IQR, 93-97\%). Sixteen patients $(52 \%)$ were subsequently prescribed morphine and midazolam as palliative care, all except one dying afterwards. Ten patients (32\%) survived and were subsequently rapidly discharged from hospital.

\section{Discussion}

HFNC is now recommended by French experts group for ICU management of patients infected with SARSCoV-2 to reduce the need for invasive mechanical ventilation (6). This technology is useful in nonhypercapnic hypoxemic pneumonia. It is a simple technique to set up and monitor. The greatest risk of HFNC might be excess mortality due to delayed intubation in case of insufficient monitoring, requiring its use in ICU (7). Because our study population was not eligible for intensive care, HFNC seemed interesting to try in our non-critical care ward.

Continuous Positive Airway Pressure (CPAP) was previously used in COVID-19 patients in UK hospitals due to the rapidly increasing number of critically ill patients and intensive care shortage (8). Patients were 
younger than our population (median age 52 ) and over half of them (14/24 (58\%)) did not subsequently require intubation. Another study which compared CPAP to standard oxygen therapy (including HFNC) for patients who were not suitable for ICU showed an extremely high mortality in both groups (9). A clinical trial is still ongoing to identify which respiratory support may be more beneficial for COVID-19 patients between three ventilation methods: CPAP, HFNC and standard treatment which involves oxygen delivered via a normal face mask or tubes in the nose (10).

The main limit of our study is its observational design without randomization between different oxygen delivery strategies. Few numbers prevent us to make comparison between people who died and people who survived. A previous study in the same hospital during the first wave showed that among 296 hospitalized patients, 30 of them older than 70 (median age 83, IQR 79-86), were treated with corticosteroids and required oxygen support for at least $9 \mathrm{~L} / \mathrm{mn}$, among whom 9 (30\%) survived. When necessary oxygen support was $12 \mathrm{~L} / \mathrm{mn}$ or more, $6 / 25$ patients $(24 \%)$ treated with corticosteroids survived (11). Our patients were older (median age 88, IQR 82-91) than people from this previous study. Further studies with careful selection of patients using the Clinical Frailty Scale (12) could help to better identify those who might benefit from treatment with HFNC.

High-flow oxygen therapy is also associated with better comfort and oxygenation than standard oxygen therapy delivered through a facemask, with a reduction in the severity of dyspnea and a decreased respiratory rate. These findings might result from the heating and humidification of inspired gas, which prevents thick secretions and subsequent atelectasis but also from low levels of PEEP (positive endexpiratory pressure) generated by a high gas flow rate and flushing of upper-airway dead space (7). This increased comfort might explain why palliative therapeutic were of little need in our study. This element of comfort is also an important help for the emotional adaptation required for the health care workers as management of death and its conditions appear as a major and central difficulty, reducing emotional fatigue, depression, anxiety and stress (13).

Indication for starting HFNC for COVID-19 patients was decided based on clinical experience. At the beginning of the wave in September 2020, we waited until oxygen standard flow reached $15 \mathrm{~L} / \mathrm{min}$ to set up HFNC. We subsequently tended to propose HFNC at earlier stage when oxygen needs increased rapidly and/or when respiratory rate remained high ( $>25 \mathrm{cycles} / \mathrm{mn}$ ) despite oxygen support, because we hypothesized that an earlier initiation could lead to better results before respiratory exhaustion. Indeed, in the sub-group of patients initiating HFNC as soon as oxygen standard flow reached $9 \mathrm{~L} / \mathrm{min}$, survival rate increased from $32 \%(10 / 31)$ to $67 \%(10 / 15)$.

\section{Conclusions}

Our data suggest that HFNC can be a successful treatment strategy in critically ill old patients with nonhypercapnic hypoxemic pneumonia due to COVID-19, and that it could be safely used outside the critical care environment for people not eligible for intensive care. Such oxygen support may be beneficial in resource-constrained countries where ventilators and intensive care resources are scarce to better cope 
with the pandemic. Clinical trials are needed to guide clinical recommendations such as optimum timing of HFNC initiation and selection of patients who would most likely benefit from it.

\section{Abbreviations}

- ICU: Intensive care unit

- HFNC: High flow nasal cannula

- RT-PCR: Reverse transcriptase polymerase chain reaction

- CT: Computed tomography

- CFS: Clinical frailty scale

- IL: Interleukine

- IQR: Interquartile range

- CPAP: Continuous positive airway pressure

- PEEP: Positive end-expiratory pressure

\section{Declarations}

\section{Ethical Approval and Consent to participate}

Our study is a non-interventional research, Assistance Publique-Hôpitaux de Paris, allows the use of routinely collected data for this using. (https://www.aphp.fr/protection-des-donnees-personnelles).

\section{Consent for publication}

Not applicable

\section{Availability of supporting data}

The datasets used and/or analysed during the current study are available from the corresponding author on reasonable request.

\section{Competing interests}

The authors declare that they have no competing interests

\section{Funding}

Not applicable

\section{Authors' contributions}

LB: 
- Conceived and designed the analysis

- Collected data

- Contributed data or analysis tools

- Performed the analysis

- Wrote the paper

VD:

- Final approval of the version to be published

BD:

- Final approval of the version to be published

SR:

- Final approval of the version to be published

DC:

- Final approval of the version to be published

GCC:

- Final approval of the version to be published

DPF:

- Final approval of the version to be published

\section{MM:}

- Final approval of the version to be published

GC:

- Final approval of the version to be published

AS:

- Conceived and designed the analysis

- Contributed data or analysis tools

- Wrote the paper

- Final approval of the version to be published 
Acknowledgements

Not applicable

\section{References}

1. Lefrancq N, Paireau J, Hozé N, Courtejoie N, Yazdanpanah Y, Bouadma L, et al. Evolution of outcomes for patients hospitalised during the first 9 months of the SARS-CoV-2 pandemic in France: A retrospective national surveillance data analysis. Lancet Reg Health - Eur. juin 2021;5:100087.

2. Docherty AB, Harrison EM, Green CA, Hardwick HE, Pius R, Norman L, et al. Features of 20133 UK patients in hospital with covid-19 using the ISARIC WHO Clinical Characterisation Protocol: prospective observational cohort study. BMJ. 22 mai 2020;369:m1985.

3. Abraham P, Courvoisier DS, Annweiler C, Lenoir C, Millien T, Dalmaz F, et al. Validation of the clinical frailty score (CFS) in French language. BMC Geriatr. 21 nov 2019;19(1):322.

4. Dexamethasone in Hospitalized Patients with Covid-19. N Engl J Med. 25 févr 2021;384(8):693-704.

5. Interleukin-6 Receptor Antagonists in Critically III Patients with Covid-19. N Engl J Med. 22 avr 2021;384(16):1491-502.

6. SRLF-SFAR-GFRUP-SPILF-SPLF-SFMU. Recommandations d'experts portant sur la prise en charge en réanimation des patients infectés à SARS-CoV2 [Internet]. 2020. Available on: https://sfar.org/download/recommandations-dexperts-portant-sur-la-prise-en-charge-en-reanimationdes-patients-en-periode-depidemie-a-sars-cov2/? wpdmdl=25387\& refresh=60f8360da9be71626879501

7. Frat J-P, Thille AW, Mercat A, Girault C, Ragot S, Perbet S, et al. High-flow oxygen through nasal cannula in acute hypoxemic respiratory failure. N Engl J Med. 4 juin 2015;372(23):2185-96.

8. Nightingale R, Nwosu N, Kutubudin F, Fletcher T, Lewis J, Frost F, et al. Is continuous positive airway pressure (CPAP) a new standard of care for type 1 respiratory failure in COVID-19 patients? A retrospective observational study of a dedicated COVID-19 CPAP service. BMJ Open Respir Res. juill 2020;7(1):e000639.

9. Aung H, Avraam E, Ashraf M, Karim N, Kiran S, Naeem M, et al. Outcomes of Continuous Positive Airway Pressure in the Management of Patients with Coronavirus (COVID-19) Pneumonia who are not Suitable for Invasive Ventilation. Open Respir Med J. 18 juin 2021;15:23-7.

10. RECOVERY-RS Respiratory Support: [Internet]. [cité 22 juill 2021]. Available on: https://warwick.ac.uk/fac/sci/med/research/ctu/trials/recovery-rs/

11. Beaumont AL, Vignes D, Sterpu R, Bussonne G, Kansau I, Pignon C, et al. Factors associated with hospital admission and adverse outcome for COVID-19: role of social factors and medical care. ECCMID; 2021.

12. Jung $\mathrm{C}$, Flaatten $\mathrm{H}$, Fjølner J, Bruno RR, Wernly B, Artigas $A$, et al. The impact of frailty on survival in elderly intensive care patients with COVID-19: the COVIP study. Crit Care. 19 avr 2021;25(1):149. 
13. Chahraoui K, Bioy A, Cras E, Gilles F, Laurent A, Valache B, et al. Psychological experience of health care professionals in intensive care unit: a qualitative and exploratory study. Ann Fr Anesth Reanim. avr 2011;30(4):342-8.

\section{Table}


Table.

Characteristics and outcome at the study population

\begin{tabular}{|c|c|c|c|}
\hline & $\begin{array}{l}\text { Overall } \\
N=31\end{array}$ & $\begin{array}{l}\text { Patients who survived } \\
N=10\end{array}$ & $\begin{array}{l}\text { Patients who } \\
\text { died } \\
N=21\end{array}$ \\
\hline \multicolumn{4}{|l|}{ Demographic characteristics } \\
\hline Age & $88(82-91)$ & $83(81-89)$ & $88(85-91)$ \\
\hline Male sex & $16(51.6)$ & $3(30.0)$ & $13(61.9)$ \\
\hline Body mass index $\left(\mathrm{kg} / \mathrm{m}^{2}\right)$ & $25(22-29)$ & $26(23-30)$ & $25(22-27)$ \\
\hline Clinical Frailty Scale & $5(3-6)$ & $4(2-5)$ & $5(4-6)$ \\
\hline \multicolumn{4}{|l|}{ Comorbidities } \\
\hline Hypertension & $20(64.5)$ & $9(90.0)$ & $11(52.4)$ \\
\hline Diabetes & $7(22.6)$ & $3(30.0)$ & $4(19.0)$ \\
\hline Heart Disease & $16(51.6)$ & $4(40.0)$ & $12(57.1)$ \\
\hline Solid cancer & $7(22.6)$ & $3(30.0)$ & $4(19.0)$ \\
\hline Other & $10(32.2)$ & $4(40.0)$ & $6(28.6)$ \\
\hline Numbers of comorbidities/patients & $2(1-3)$ & $2(2-3)$ & $3(1-3)$ \\
\hline \multicolumn{4}{|l|}{ Chest CT scan lesions } \\
\hline None, minimal or moderate $(<25 \%)$ & $16(51.6)$ & $4(40.0)$ & $12(57.1)$ \\
\hline Extensive (25-49\%) & $9(29.0)$ & $4(40.0)$ & $5(23.8)$ \\
\hline Serious or critical (>50\%) & $6(19.4)$ & $2(20.0)$ & $4(19.0)$ \\
\hline \multicolumn{4}{|l|}{ Characteristics at HFNC initiation } \\
\hline Time since hospital admission (days) & $3(1-6)$ & $4(2-5)$ & $2(1-6)$ \\
\hline Saturation on oxygen therapy (\%) & $95(93-97)$ & $96(94-98)$ & $94(93-97)$ \\
\hline Oxygen flow, L/min ${ }^{-1}$ & $12(8-15)$ & $9(7-9)$ & $12(9-15)$ \\
\hline \multicolumn{4}{|l|}{ Outcomes } \\
\hline Weaned off HFNC and discharged & $10(32)$ & - & - \\
\hline Duration of HFNC therapy, days & $6(4-10)$ & $10(7-12)$ & $5(4-7)$ \\
\hline Hospitalisation duration, days & $13(8-22)$ & $22(17-24)$ & $9(5-13)$ \\
\hline
\end{tabular}


Data are presented as medians (IQR) or $\mathrm{n}(\%)$

IQR, Interquartile range; CT, computed tomography; HFNC, High flow nasal cannula oxygen.

\section{Supplementary Files}

This is a list of supplementary files associated with this preprint. Click to download.

- SupplementaryTable.docx 